Journal of Patient-Centered

\title{
Advancing Population Health: New Models and the Role of Research. An Overview of the 22nd Annual Health Care Systems Research Network Conference
}

Robert T. Greenlee

Lois E. Lamerato

Sarah M. Greene

Follow this and additional works at: https://aah.org/jpcrr

Part of the Clinical Epidemiology Commons, Health Information Technology Commons, Health Services Research Commons, and the Translational Medical Research Commons

\section{Recommended Citation}

Greenlee RT, Lamerato LE, Greene SM. Advancing population health: new models and the role of research. An overview of the 22nd annual Health Care Systems Research Network Conference. J Patient Cent Res Rev. 2016;3:155-8. doi: 10.17294/2330-0698.1284

Published quarterly by Midwest-based health system Advocate Aurora Health and indexed in PubMed Central, the Journal of Patient-Centered Research and Reviews (JPCRR) is an open access, peer-reviewed medical journal focused on disseminating scholarly works devoted to improving patient-centered care practices, health outcomes, and the patient experience. 


\title{
Advancing Population Health: New Models and the Role of Research. An Overview of the 22nd Annual Health Care Systems Research Network Conference
}

\author{
Robert T. Greenlee, PhD, MPH, ${ }^{1}$ Lois E. Lamerato, $\mathrm{PhD},{ }^{2}$ Sarah M. Greene, $\mathrm{MPH}^{3}$ \\ ${ }^{1}$ Center for Clinical Epidemiology and Population Health, Marshfield Clinic Research Foundation, Marshfield, WI \\ ${ }^{2}$ Department of Public Health Sciences, Henry Ford Health System, Detroit, MI \\ ${ }^{3}$ Health Care Systems Research Network, Seattle, WA
}

\begin{abstract}
The Health Care Systems Research Network (formerly HMO Research Network) held its annual conference in Atlanta, Georgia, in April 2016. The conference, with a theme of "Advancing Population Health: New Models and the Role of Research," was co-hosted by Marshfield Clinic Research Foundation and Henry Ford Health System's Department of Public Health Sciences. Key sessions reflecting the theme included plenary panels with a research focus on patient-centered care and population health management, a full-day symposium on precision oncology and a half-day patientengaged research training workshop. (J Patient Cent Res Rev. 2016;3:155-158.)
\end{abstract}

Keywords HCSRN; health care research; integrated delivery; managed care programs; multicenter study

Established in 1994 (as the HMO Research Network), the Health Care Systems Research Network (HCSRN) is a consortium of 20 research organizations embedded within, or affiliated with, integrated and other large health care delivery systems in the United States and Israel. ${ }^{1}$ The stated mission of the network is to improve individual and population health through research that connects the resources and capabilities of learning health care systems. ${ }^{2}$ More than 1,900 embedded investigators and research staff work within, across and beyond HCSRN member organizations to support and conduct a wide array of health research, including epidemiology, health services, health economics, behavioral health, genomics, health equity and more.

Collaborations in the HCSRN are facilitated by a common commitment to public domain research, sharing scientific interests and best practices, creating standardized data models with high capture of health care claims and electronic medical record data for defined population cohorts of more than 15 million

Correspondence: Robert T. Greenlee, PhD, MPH,

Marshfield Clinic Research Foundation, 1000 N. Oak Avenue, Mail Stop ML2, Marshfield, WI, 54449, T: 715-389-3537,

F: 715-389-3880, Email: greenlee.robert@mcrf.mfldclin.edu health plan members and patients, ${ }^{3}$ and developing common efficiencies in research support and administration. Using these critical capacities, the HCSRN has a successful history of establishing funded research consortia focused on "real-world" populations in the context of care delivery across a wide range of topic areas, including cancer, cardiovascular disease, medical product safety, diabetes, mental health, aging, substance abuse and others. ${ }^{1}$

The HCSRN has convened an annual conference since 1995 for researchers, study staff, potential collaborators, research funders and other stakeholders to gather and share their best science and confer to establish and foster the research of the future. ${ }^{4}$ Published intermittently in early years, annual meeting proceedings from the HCSRN have been published continuously since 2008. ${ }^{5,6}$ The 2016 HCSRN conference was held April 13-16 in Atlanta, Georgia, with Marshfield Clinic Research Foundation (Marshfield, WI) and the Department of Public Health Sciences at Henry Ford Health System (Detroit, MI) serving as scientific cohosts. The conference planning committee, which included additional representation from other HCSRN member sites and from central HCSRN leadership, selected the conference theme "Advancing Population Health: New Models and the Role of Research" to 
explore and highlight new approaches that research groups and health care systems are applying to improve the health of their patients and communities.

To support the 2016 conference theme, threads of population health and patient-centeredness were intentionally woven through the invited components of the three-day scientific agenda. The morning plenary on Day 1 focused on patient-centered care and the important role of research, with Mary Durham, $\mathrm{PhD}$, director of the Center for Health Research at Kaiser Permanente Northwest (Portland, OR), serving as the moderator and discussant. Panelists from Group Health Research Institute (Seattle, WA), Kaiser Permanente Northern California (Oakland, CA) and Medica Research Institute (Minnetonka, MN) presented innovative work in this area, including:

- qualitative research defining and improving assessment of patient-centeredness from a variety of stakeholder perspectives;

- a broad initiative pursuing stakeholder engagement on translational research in diabetes; and

- use of parent peer mentors to increase insurance coverage among previously uninsured children.

The morning plenary session on Day 2 focused on new approaches to manage the health of patient and community populations. Eric Larson, MD, MPH, executive director of Group Health Research Institute, moderated the session, and Steve Clauser, PhD, MPA, program director of the Improving Healthcare Systems Research Program at the Patient-Centered Outcomes Research Institute (PCORI) was the discussant. Nationally recognized panelists represented the Veterans Affairs (VA) Center for Implementation Practice and Research Support, the Office of Health Systems Collaboration in the Centers for Disease Control and Prevention, and the Center for Health Policy and Health Services Research at Henry Ford Health System. Panel presentations included:

- the role and methods of implementation science as a key tool for realizing the promise of learning health systems;

- health policy perspectives on new models of care delivery and coverage; and

- a new collaboration between public health and the private health care sector on prevention initiatives to improve health and control health care costs.
The conference format was adjusted in 2016 to offer more in-depth attention to selected high-profile topics through longer focused sessions, including a half-day training workshop on patient engagement in research and a full-day symposium on precision oncology. The workshop was presented by a multisite collaborative team from HCSRN's Patient Engagement in Research Scientific Interest Group. (To learn more about patient-engaged research activities, please see the article by Madrid and colleagues summarizing their timely workshop, also published in this conference proceedings supplement to the Journal of PatientCentered Research and Reviews. ${ }^{7}$ )

The precision oncology symposium was presented jointly on behalf of the HCSRN conference by the Cancer Research Network and the American Cancer Society, with additional partners on the planning group from the National Cancer Institute, Centers for Disease Control and Prevention and Emory University. It included four sessions, the first of which comprised a morning keynote from Deborah Bruner, $\mathrm{RN}, \mathrm{PhD}$, Woodruff Professor of Nursing at the Emory Winship Cancer Institute, along with a panel of moderators/ discussants from the American Cancer Society, National Cancer Institute and Kaiser Permanente. The second session focused on innovative examples of health system integration of genomics, oncology and patient-centeredness from Geisinger Health System (Danville, PA), Kaiser Permanente Northwest, Henry Ford Health System and the VA. The luncheon plenary keynote speaker was Joe Selby, MD, MPH, executive director of PCORI, with a reaction panel of patient partner advisors and patient-engaged researchers. The final session in the afternoon was a well-attended working meeting, with stage-setting presentations followed by roundtable discussions to develop nearterm priorities for research on precision medicine in cancer within the Cancer Research Network and the HCSRN. Concurrently on the conference's final day, Virtual Data Warehouse Implementation Group members held a working session to share best practices and inform future directions with regard to developing new data content areas for current and emerging research priorities.

The 2016 State of the Network plenary session celebrated both the "history" and "future" of the 
network. Jerry Gurwitz, MD, executive director of the Meyers Primary Care Institute (Worchester, MA) and chair of the HCSRN Governing Board, provided the annual update on the status of recent network leadership discussions, but reserved the majority of the session to gain insights from leaders of the two newest HCSRN member organizations, Catholic Health Initiatives (Englewood, CO) and Medica Research Institute, as well as from HCSRN's inaugural executive director, Sarah Greene, MPH. Dr. Gurwitz and Ms. Greene described the importance of evolving the HCSRN's capabilities, membership and data resources in response to changes in the broader research environment. Special attention also was given this year to recognize and appreciate the longstanding service and commitment from two outgoing governing board members, Mary Durham, $\mathrm{PhD}$, representing Kaiser Permanente Northwest and Hawaii, and Mark Hornbrook, $\mathrm{PhD}$, representing Kaiser Permanente Northwest.

The 2016 conference maintained its longstanding framework of highlighting emerging scientists in the network. An afternoon plenary session featured winners of the 2016 Early Career Investigator awards, who presented the following diverse topics:

- effect of clinical practice guideline changes on prostate cancer screening;

- effect of California's pay-for-performance program on adolescent human papilloma virus (HPV) vaccination;

- the relation between provider-patient racial/ethnic concordance and patient-reported experience; and

- a patient-centered, pharmacist-led approach to lipid management via reporting genomic testing results to patients with familial hypercholesterolemia.

This session was capped off with presentation of the first-ever HCSRN Mentor of the Year Award, a welldeserved honor given to John Steiner, MD, MPH, senior director of the Institute for Health Research at Kaiser Permanente Colorado (Denver, CO).

The conference agenda also incorporated 40 ancillary meetings in which new and existing project teams and scientific interest groups met to establish and foster collaborative endeavors. For example, a working meeting explored the value of mentoring in the network and began a formal conversation to promote a formal mentoring program in the HCSRN. Also, two special submitted panel sessions were organized: one by the Virtual Data Warehouse Operations Committee on multisite data and collaboration, and one by representatives from PCORI on differences in comparative effectiveness research between fee-forservice and integrated health systems.

Lastly, nearly 130 peer-reviewed scientific abstracts provided insights into the support, conduct and results of research across 13 podium and two poster sessions. Topic categories included: chronic conditions; health services, health policy and cost; health informatics and big data; aging and multimorbidity; genetics, genomics and precision medicine; cancer; care improvement; communications, dissemination and implementation; health disparities; mental health, alcohol and substance use; and patient, provider and health system engagement. A selection of the abstracts presented at the conference in oral and poster format appears elsewhere in this issue supplement. ${ }^{8}$

For readers who would like to learn more about the HCSRN and its annual conference, the HCSRN website, www.hcsrn.org, provides a range of informative links, not only to conference materials and presentations from the 2016 meeting and other past conferences, but also to pages featuring the funded networks and major research initiatives within the HCSRN and to a variety of collaborative tools and support materials. Finally, those interested should save the dates for next year's annual conference, to be hosted by Sutter Health/Palo Alto Medical Foundation (Palo Alto, CA) in San Diego, California, March 20-23, 2017. The call for abstracts opens in August 2016 at www.hesrnmeeting.org.

\section{Acknowledgments}

The authors would like to acknowledge the work of the 2016 HCSRN planning committee, the planning groups for the patient-engaged research workshop and precision oncology symposium, the conference management team at JBS \& Associates, the central HCSRN leadership team, and this year's financial sponsors - American Cancer Society, the National Cancer Institute-sponsored Cancer Research Network, Marshfield Clinic Research Foundation and Henry Ford Health System's Department of Public Health Sciences. Importantly, we acknowledge and appreciate 
the hard work and dedication of the conference cocoordinators, Deb Multerer from Marshfield Clinic Research Foundation and Heather Lipkovich from Henry Ford Health System; the conference would not have been possible without them.

\section{References}

1. Steiner JF, Paolino AR, Thompson EE, Larson EB. Sustaining research networks: the twenty-year experience of the HMO Research Network. EGEMS (Wash DC). 2014;2:1067. CrossRef

2. Health Care Systems Research Network home page. www. hcsrn.org. Accessed June 20, 2016.

3. Ross TR, Ng D, Brown JS, Pardee R, Hornbrook MC, Hart G, Steiner JF. The HMO Research Network Virtual Data Warehouse: a public data model to support collaboration. EGEMS (Wash DC). 2014;2:1049. CrossRef

4. Thompson EE, Steiner JF. Embedded research to improve health: the 20th annual HMO Research Network Conference, March 31-April 3, 2014, Phoenix, Arizona. Clin Med Res. 2014;12:73-6. CrossRef
5. Greenlee RT, Coleman LA, Nelson AF, Selby JV. Partnerships in translation: advancing research and clinical care: the 14th annual HMO Research Network Conference, April 13-16, 2008, Minneapolis, Minnesota. Clin Med Res. 2008;6:109-12. CrossRef

6. Gould MK. Partnering to improve care: highlights from the 21st annual conference of the HMO Research Network. $J$ Patient Cent Res Rev. 2015;2:75-7. CrossRef

7. Madrid S. Tuzzio L, Stults C, Wright LA, Napolitano G, Dillon E, Tabano H, Greene SM. Sharing experiences and expertise: the Health Care Systems Research Network workshop on patient engagement in research. J Patient Cent Res Rev. 2016;3:159-66.

8. Abstracts from the 22nd annual Health Care Systems Research Network Conference, April 13-16, 2016, Atlanta, Georgia. J Patient Cent Res Rev. 2016;3:167-228.

(C) 2016 Aurora Health Care, Inc. 